\title{
The Diffusion of Cholera in Egypt, 1947: A Time-Space Analysis of One of the Largest Single Outbreaks in the Twentieth Century
}

\author{
Matthew Smallman-Raynor * and Andrew D. Cliff ${ }^{\dagger}$
}

\section{$\underline{\text { Affiliations }}$}

*School of Geography, University of Nottingham, University Park, Nottingham, NG7 2RD, U.K.

†Department of Geography, University of Cambridge, Downing Place, Cambridge, CB2 3EN, U.K.

Corresponding author

Matthew Smallman-Raynor, School of Geography, University of Nottingham, University Park, Nottingham NG7 2RD, U.K.; email: matthew.smallman-raynor@nottingham.ac.uk; tel: +44 (0)115 9515427; fax: +44 (0)1159515249. 


\begin{abstract}
The epidemic of cholera that spread through Egypt in the latter months of 1947 was one of the largest single outbreaks of the disease in the twentieth century. Using a swash-backwash model, this paper examines the geographical wave-like spread and subsequent retreat of the epidemic from an apparent origin in the settlements and prisoner-of-war camps of the Nile Delta area to reach its maximum geographical extent some six weeks later at Aswan $(850 \mathrm{~km}$ away). Our results demonstrate the very rapid spatial advance of the epidemic wave through the provinces and governorates of Egypt, with an approximately linear sequence of progression up the Nile. Superimposed on this national pattern are pronounced differences in the rate of epidemic advance in the traditional geographical divisions of Lower and Upper Egypt. Alternative visualisations of the cholera spaces of Egypt, using techniques of multidimensional scaling (MDS) and cluster analysis, underscore the differential patterns of cholera transmission in these areas of the country. The patterns are interpreted in relation to a vigorous control effort that included restrictions on public transport, patient isolation, contact tracing and mass vaccination of the entire population exposed to risk of infection.
\end{abstract}

$\underline{\text { Keywords }}$

Cholera; Cluster analysis; Egypt; 1947 epidemic; Spatial wave propagation; Swash-backwash model

$\underline{\text { Running head }}$

1947 cholera epidemic in Egypt 


\section{Highlights}

- Geographical transmission of the epidemic is modelled using robust spatial methods

- The epidemic spread wave-like up the Nile from the Nile Delta area

- The rate of epidemic propagation differed in the divisions of Lower and Upper Egypt

- Country-wide spread had been achieved before vaccines became available in quantity 
The Egyptian cholera epidemic of 1947 represented a stern test for the contemporary machinery of communicable disease control. ${ }^{1}$ On one level, the response to the epidemic can be viewed as an early success in post-war global health governance. ${ }^{2}$ Rallied by the Interim Commission of the World Health Organization (WHO), Egypt received almost 6 million ml of cholera vaccine from donor countries. ${ }^{3}$ China, alone, donated 2 million $\mathrm{ml}$ of vaccine, while the US military ferried in syringes and other medical paraphernalia. ${ }^{4}$ By the end of 1947, the Egyptian epidemic was all but over and Brock Chisholm, Executive Secretary of the Interim Commission, could declare the control effort to be 'an outstanding achievement of modern preventive medicine' - an achievement for which he would eventually be awarded the Anti-Cholera Memorial Medal by the Government of Egypt. ${ }^{5}$

Notwithstanding this apparent success, the Egyptian cholera epidemic of 1947 assumed the status of one of the largest single cholera outbreaks of the twentieth century. In the manner of a wave breaking on the seashore, the epidemic arrived in the Nile Delta in late September 1947 and, over the next few weeks, washed up the Nile through the settlements of the valley as far as Aswan. The epidemic then retreated like a backwash down the Nile into the delta until the last cases were recorded in Cairo at the end of December. ${ }^{6}$ Almost 21,000 cases and over 10,000 deaths were initially reported, although the actual levels of morbidity and mortality are likely to have been substantially higher. ${ }^{7}$ Internationally, the infection was at risk of being entrained in the Hajj pilgrimage crowds of 1947, and emergency measures were imposed to mitigate this risk. ${ }^{8}$

While the political repercussions of the 1947 Egyptian cholera epidemic have attracted the attention of scholars, much less attention has been paid to what Robert Stock refers to as the 'somewhat enigmatic' epidemiological features of the event. ${ }^{9}$ Foremost among the latter, Stock has alluded to the unusual rapidity with which the infection wave spread through the Egyptian population. ${ }^{10}$ To examine the geographical dimensions of this issue, the present paper draws on a robust model of epidemiological analysis (swashbackwash model) that has been specifically designed to assess the spatial velocity of epidemic advance and retreat in a defined population. ${ }^{11} \mathrm{We}$ begin our examination with a brief overview of the nature and epidemiology of cholera. Turning to the Egyptian epidemic 
of 1947, we use weekly reports of cholera cases in the (then) twenty-one provinces and governorates of Egypt to calibrate the swash-backwash model. Our results confirm the very rapid spatial advance of the epidemic wave through the provinces and governorates, with an approximately linear sequence of progression up the Nile. Superimposed on this national pattern are pronounced differences in the rate of epidemic propagation in the traditional geographical divisions of Lower and Upper Egypt. Alternative visualisations of cholera activity in Egypt, using techniques of multidimensional scaling (MDS) and cluster analysis, underscore the differential patterns of disease transmission in these areas of the country. Taken together, our results demonstrate that it was the rapid spatial velocity of the infection wave - most especially in the Nile Delta area of Lower Egypt - that permitted the epidemic to elude any early control efforts and to become widely seeded in the country.

\section{Cholera: Nature and Historical Context}

Cholera is an acute intestinal disease caused by infection with the bacterium Vibrio cholerae. The bacterium is most commonly acquired through the ingestion of contaminated water, although food may also serve as a vehicle of infection. In terms of clinical presentation, infection with $V$. cholerae ranges from asymptomatic or mild to severe. In severe cases, an incubation period of two to five days is typically followed by the sudden onset of diarrhoea and vomiting. Consequent symptoms include cramps, a reduction in body temperature and blood pressure and, ultimately, death due to dehydration, electrolyte loss and circulatory shock within a few hours or days of symptom onset. Mortality is typically witnessed in 40-60 percent of untreated patients with severe disease. For those who survive, the period of communicability of $V$. cholerae is generally for a few days after recovery. Occasionally, however, a carrier state may persist for several months. ${ }^{12}$

The epidemic transmission of cholera is closely associated with the lack of potable water, poor sanitation and hygiene and overcrowded living conditions. ${ }^{13}$ Contaminated watercourses are the most common originating sites of infection with $V$. cholerae and they are generally regarded as a prerequisite for large cholera outbreaks in newly infected (nonendemic) settings. In the context of the present study, for example, most of the population of 
Egypt in 1947 (19 million) lived in the Nile Delta or Valley areas and made use of Nile water for one purpose or another. In this way, the Nile became a critical driver of cholera spread once the disease had been introduced into the population.

In the words of Pollitzer and colleagues, the year 1817 marked 'a new epoch in the history of cholera'. ${ }^{14}$ In that year, cholera began to spread as the first in a series of six great pandemics which, over the ensuing decades of the nineteenth century, extended across much of the inhabited world. ${ }^{15}$ The sixth pandemic persisted well into the twentieth century, eventually to terminate by the mid-1920s. ${ }^{16}$ The global record of cholera mortality in the twentieth century, encompassing the long tail of the sixth pandemic (1899-1923) and the formative decades of the seventh (El Tor) pandemic (1961-present), is plotted in Fig. 1. ${ }^{17}$ Despite the fact that cholera has been covered by the International Sanitary Conventions and successor regulations since 1893 , and is therefore subject to mandatory notification, the global record of cases and deaths is incomplete. ${ }^{18}$ Subject to this data caveat, the broad trend of a century-long decline in mortality from the disease is marked, as is the shift in the geographical focus from South East Asia to Africa and the Americas since mid-century. This has continued into the twenty-first century with the Haiti outbreak in 2010 which, still ongoing, has become the largest single outbreak recorded globally since the seventh (El Tor) cholera pandemic began in $1961 .{ }^{19}$

\section{FIGURE 1 NEAR HERE}

\section{Cholera in Egypt, 1947}

Because Egypt lies at the meeting point of east and west, linked by sea, land and air to the three surrounding continents of Europe, Asia and Africa, it was repeatedly ravaged by destructive epidemics of cholera in the nineteenth century that resulted in tens of thousands of deaths. ${ }^{20}$ Into the twentieth century, a further outbreak spread in 1902 to cause a reported 40,613 cases with 34,595 deaths in over 2,000 towns and villages across Egypt. This was the last epidemic to occur in the country prior to the event of 1947 (Fig. 1). ${ }^{21}$

Estimates of cases and mortality over the three to four month duration of the 1947 epidemic vary wildly. Shousha's preliminary report gives a lower bound of 20,804 cases and 
10,277 deaths, a case-fatality rate (CFR) of almost 50 percent. $^{22}$ With the passage of time, these figures have been revised upwards to 35,000 cases with 20,000 deaths, a CFR of almost 60 percent, reminiscent of the great outbreaks of the nineteenth century. ${ }^{23}$ Apart from a possible Syrian link, the disease was confined to Egypt. Fig. 2 plots the time series of reported cases and deaths in the epidemic, while Fig. 3 shows the geographical distribution and place names.

\section{FIGURE 2 NEAR HERE}

\section{FIGURE 3 NEAR HERE}

\section{Epidemic origins and dispersal}

Like the 2010 Haiti outbreak, much speculation has surrounded its precise origins because the index case was never openly identified by the World Health Organization (WHO) ${ }^{24}$ The evidence is reviewed at length by Gallagher. ${ }^{25}$ The country may have been the first to fall victim to cholera as a result of air travel of infected persons. Prior to 1947 , cholera commonly made its way into Egypt via returning pilgrims from Mecca (Saudi Arabia), resulting in major outbreaks that usually began in the Cairo-Alexandria area. But, in the 1947 outbreak, the index cases are thought to be have been imported from India by British military personnel (Fig. 4). As Gallagher notes, in September 1947 the only significant outbreak of cholera which was ongoing globally was in the Punjab region of India. ${ }^{26}$ British colonial India had just become independent and the new state of Pakistan was being formed from the western provinces of the former colony. Communal strife had led to the mass flight of Muslim and Hindu refugees. In early September cholera appeared among these refugees, while simultaneously thousands of British troops were being evacuated from India after the end of the Second World War. ${ }^{27}$ The stop-over location on the way home was Egypt's Tal alKabir/al-Qurayn military base and airfield complex near Cairo (Fig. 3). Local labourers billeted in al-Qurayn were employed to service the needs of the various bases and camps in the area, including some 6,000 workers attached to the British airfield. Many of the early victims were among these labourers. ${ }^{28}$ To add to the general confusion, some 100,000 
German prisoners of war who were awaiting repatriation were detained in labour camps in Egypt, mainly in the Nile Delta region. Among the camp locations was Tal al-Kabir.

\section{FIGURE 4 NEAR HERE}

Whatever the epidemic's origin, al-Qurayn was an excellent diffusion pole because thousands of merchants from all over Egypt and elsewhere had gathered there for the annual date fair when the first cases of cholera appeared. ${ }^{29}$ From this index location, cholera reached Cairo in late September and then continued on to Ismailia before reaching all the provinces of Lower Egypt. By October, it reached Upper Egypt, albeit with limited impact. The geographical spread of the epidemic up the Nile is mapped schematically by week of report in Fig. 5. The epidemic waned after November and petered out in December with the onset of winter.

\section{FIGURE 5 NEAR HERE}

\section{Modelling geographical spread: data sources}

To examine the geographical spread of the 1947 cholera epidemic from its putative origin in the vicinity of al-Qurayn, we draw on epidemiological and demographic information included in Sir Aly Tewfik Shousha's report to the WHO. ${ }^{30}$ Acting in his capacity as Under-Secretary of State in the Egyptian Ministry of Health, and vice chairman of the WHO Interim Commission, Shousha's report provides a summary overview of the sanitary and administrative measures implemented in the course of the cholera epidemic, along with information on scientific aspects of the disease and its documented occurrence. For each of the 20 provinces and governorates mapped in Fig. 3, and an additional area known as Frontiers (a collective term for an amalgam of areas that include Sinai and the desert areas to the west and east of the Nile), the report includes information on: (i) the weekly count of recorded cases and deaths from cholera for the duration of the epidemic (weeks 39-52 of 1947); and (ii) associated population estimates of the provinces and governorates. These data were used to form 14 (epidemic weeks) $\times 21$ (geographical units) time-space matrices of 
cholera cases, deaths and associated rates per 100,000 population. The resulting matrices of cholera counts and rates form the basis of all subsequent analysis.

\section{Spread models, I: the swash-backwash model}

To analyse the temporal rate and geographical reach of cholera during the 1947 epidemic, the swash-backwash model of spatial epidemiological analysis was used. ${ }^{31}$ The model measures the rate of spatial advance and retreat of an infection wave across a set of geographical units (here the provinces and governorates of Egypt). Egypt provides a particularly interesting spatial setting in which to apply the model because the governorates and provinces are distributed linearly along the Nile Valley and Delta which, as discussed earlier, was a principal driver of cholera transmission in the outbreak.

\section{$\underline{\text { Methods }}$}

Full details of the swash-backwash model, including a derivation of the key measures of the model, are provided elsewhere. ${ }^{32}$ We limit our consideration here to the operationalisation of the model in relation to the 1947 cholera epidemic. In brief, the first week of the epidemic was coded $t=1$ (week 39 of 1947) and the subsequent weeks were coded serially as $t=2$, $3, \ldots, 14$ (week 52, the last week of the epidemic). For each province/governorate, we refer to the first week in which a case of cholera was reported as the leading edge (LE) and the last week in which a case was reported as the following edge $(F E)$ of the epidemic in that geographical unit. The $L E$ and $F E$ were then used to compute the following series of measures of spatial velocity: ${ }^{33}$

(1) Average velocity ratios $\left(V_{L E}, V_{F E}\right)$. The average time in weeks of arrival $(L E)$ and departure $(F E)$ of the infection wave across the set of provinces/governorates was computed as a time-weighted arithmetic mean, $\bar{t}_{L E}$ and $\bar{t}_{F E}$. These means were then converted to dimensionless velocity ratios $V_{L E}$ and $V_{F E}$ with values in the range $[0,1]$.

(2) Integrals $\left(\boldsymbol{S}_{A}, \boldsymbol{I}_{A}, \boldsymbol{R}_{A}\right)$. A time-space map of the outbreak was defined according to the number of areas which, in a given week, were in each of three states: (i) unaffected by cholera (susceptible, S), having not reported any cases of the disease; (ii) affected by cholera (infected, I), with cases of the disease being reported; and (iii) formerly affected 
by cholera (recovered, $R$ ), with the outbreak having passed through and cases of the disease no longer being reported. The total amount of the time-space map (the integrals $S_{A}, I_{A}, R_{A}$ ) in which the provinces/governorates of Egypt were in the states $S, I$ and $R$ was then calculated to obtain an overview of the outbreak. All three integrals are dimensionless numbers with values in the range $[0,1]$.

(3) Spatial basic reproduction rate $\left(R_{0 A}\right)$. To provide a measure of the propensity of an infected geographical unit to spawn other infected units in later time periods, the spatial basic reproduction rate, $R_{0 A}$, of the epidemic was derived from the integrals $S_{A}$ and $R_{A}$

Summary descriptors for velocity measures (1)-(3) are provided in Table 1. In essence, the measures provide an indication of the rate of advance and retreat of the epidemic wave:

(1) Epidemic advance. The relatively fast advance of an epidemic wave is denoted by relatively high values of $V_{L E}$ and low values of $S_{A}$. Conversely, the relatively slow advance of an epidemic wave is denoted by relatively low values of $V_{L E}$ and high values of $S_{A}$;

(2) Epidemic retreat. The relatively fast retreat of an epidemic wave is denoted by relatively high values of $V_{F E}$ and high values of $R_{A}$. Conversely, the relatively slow retreat of an epidemic wave is denoted by relatively low values of $V_{L E}$ and low values of $R_{A}$

In addition, $I_{A}$ provides a measure of the relative rate of spatial advance and retreat while $R_{0 A}$ calibrates the spatial velocity of disease spread, with higher values denoting more rapid spatial propagation (Table 1).

\section{TABLE 1 NEAR HERE}

\section{$\underline{\text { Results }}$}

Figs. 6 and 7 summarise the results of the analysis. Fig. 6A shows the susceptible $(S)$, infected $(I)$ and recovered $(R)$ states of each province and governorate by week of the 
epidemic, while Figs. 6B and $\mathrm{C}$ use vectors to trace the spatial course of the infection $(I)$ and recovery $(R)$ processes. In terms of the infection process, Fig. 6B shows that the epidemic progressed almost linearly up the Nile over a period of 14 weeks from its initial appearance at al-Qurayn (Cairo, governorate, 1), to peter out before Aswan (20). The recovery process, summarised in map $(\mathrm{C})$, shows that province/governorate recovery was more spatially erratic than infection, with several long distance leaps between Upper and Lower Egypt. The integrals in (D) indicate that nearly 60 percent of the space-time map was in the infected state, substantially more than either the susceptible or recovered states. In addition, the susceptible state is much the smallest of the three integrals. Taken together, these results imply a widespread epidemic which diffused very rapidly across Egypt.

\section{FIGURE 6 NEAR HERE}

Fig. 7 plots various model parameters which characterise the nature and velocity of the epidemic. First, the lower graph in part (A) plots the number of provinces and governorates which were newly infected with cholera in each week of the epidemic (epidemic expansion or 'swash' phase) and the number in which cholera activity had newly terminated (epidemic retreat or 'backwash' phase). The graph shows an imbalance in time between the swash (weeks 39-43) and backwash phases (weeks 44-51), with a front-end loading of cases in the swash phase; 88 percent of recorded cases had occurred by week 44 .

\section{FIGURE 7 NEAR HERE}

Fig. 7B plots the time curves for the $S_{A}, I_{A}, R_{A}$ integrals. The curves are shown for the provinces and governorates of both Lower Egypt and Upper Egypt and for Egypt as a whole. The curves complement the findings of Fig. 6 by confirming that the susceptible state as defined by the $S_{A}$ curves was of much smaller extent (that is, susceptible provinces/governorates became infected much faster) in Lower than Upper Egypt. Similarly, the infected state as defined by the $I_{A}$ curves was much more extensive (that is, cholera was present for much longer in provinces/governorates) in Lower than Upper Egypt.

Finally, Fig. 7C shows that both the leading edge velocity, $V_{L E}$, and the spatial reproduction rate, $R_{0 A}$, were substantially greater in Lower than Upper Egypt. This 
underscores the much more rapid spatial transmission process for the epidemic in the provinces/governorates of Lower Egypt.

Taken together, a clear picture emerges from Figs. 6 and 7 of an epidemic which spread rapidly and extensively in the Nile Delta before progressing more slowly up the Nile into the provinces and governorates of Upper Egypt.

\section{Spread models, II: Egypt's cholera spaces}

To cross-check the results from the swash-backwash model, the time series of reported case and death rates for the 21 governorates and provinces were analyzed using multidimensional scaling (MDS) and cluster analysis.

\section{$\underline{\text { Methods }}$}

MDS refers to a family of statistical methods by which information contained in a data set is represented by the configuration of points in multidimensional space, such that the geometrical relationships between the points reflect the empirical relationships in the data. ${ }^{34}$ If the space so constructed is in two dimensions, the resulting configuration is map-like.

The concepts which underpin MDS mapping are straightforward. On a conventional geographical map, the relative locations of points correspond to their (scaled) geographical locations. In MDS mapping, the relative locations correspond instead to their degree of similarity on some variable measured for them. For example, we might map points into a time space; locations separated by short travel times will appear close together in such a space, while centres linked by long travel times will be widely separated. In general terms, the greater the degree of similarity between places on the variable measured, the closer together the places will be in the MDS space. Conversely points which are dissimilar on the variable will be widely separated in the MDS space, irrespective of their geographical locations on a conventional map. The MDS problem is to find a configuration of $n$ points in $m$-dimensional space such that the inter-point distances in the configuration match the experimental (dis)similarities of the $n$ objects as accurately as possible. Accuracy is determined from the stress coefficient. Low values of the stress coefficient imply a good fit of the model while high values of the stress coefficient imply a poor fit. 
Model fitting was conducted using the Pearson correlation coefficient to measure similarity between the cholera time series. Analysis of both case and death rates per 100,000 population was carried out. Within the cholera spaces, governorates and provinces which are similar in terms of their cholera notification rates will appear close together, irrespective of their geographical locations on the map of Egypt. Conversely, governorates and provinces that are dissimilar in terms of their cholera rates will appear widely separated, even though they may be geographically proximal. In all instances, MDS solutions were seeded by the longitude/latitude $(x, y)$ co-ordinates of the geographical centroids of the governorates and provinces, with the Pearson product moment correlation coefficient, $r$, forming the measure of similarity.

To supplement the MDS analysis, the weekly time series of case and death rates per 100,000 population for the set of provinces/governorates were subjected to techniques of cluster analysis. The principles of cluster analysis are summarised by Everitt and colleagues. ${ }^{35}$ As applied here, the approach involves the grouping of provinces/governorates in such a way that intra-group similarities in cholera rates are greater than inter-group similarities. For the purposes of the present analysis, similarities were defined by the Pearson correlation coefficient between the time series of cholera activity for each pair of provinces/governorates, with the application of a hierarchical (complete linkage) algorithm used to form the resulting clustering dendrograms in Figure $8 .{ }^{36}$

\section{$\underline{\text { Results }}$}

Figure 8 shows the two-dimensional minimum stress MDS configurations (left) and the dendrograms for the complete linkage cluster analysis (right) for (A) case rates and (B) death rates. Echoing the findings of the swash-backwash model, the striking feature of Fig. 8 is the primary separation of the provinces and governorates of Upper and Lower Egypt. The MDS configurations reveal the generally similar patterns of cholera activity in the Nile Delta / Suez Canal area of Lower Egypt while, at another level, the cluster analysis separates the governorates and provinces of the Nile Delta into east and west. 


\section{Discussion}

In his book Cholera in Africa, Stock described the unusually rapid dissemination of cholera in Egypt as one of the enigmas of the 1947 epidemic. ${ }^{37}$ To further an understanding of this aspect of the epidemic, we have applied the swash-backwash model of spatial epidemiological analysis to weekly reports of cholera activity in 21 provinces and governorates of the country. Our analysis yields the following insights into the spatial propagation of the infection wave:

(1) from an apparent introduction of $V$. cholerae in the vicinity of the town of al-Qurayn in late September, the disease advanced with an approximately linear sequence of progression up the Nile (Figs. 5 and 6B);

(2) the main phase of spatial wave propagation continued for five weeks until late October (swash stage), waning thereafter (backwash stage) (Figs. 6A and 7A);

(3) estimated from the integrals of the swash-backwash model (Figure 6D), the mean duration of the principal phases of the epidemic in a given province/governorate was two weeks (susceptible phase), eight weeks (infective phase) and four weeks (recovered phase), with the low estimate for the susceptible phase underscoring the very rapid spatial colonisation of the infection wave; and

(4) when examined in terms of the historic divisions of Egypt, the infection wave advanced much faster and persisted longer in Lower than Upper Egypt (Fig. 7B, C).

The similarities in patterns of wave propagation identified by the swash-backwash model for the provinces/governorates of Lower Egypt are underscored by the two-dimensional MDS configurations and cluster dendrograms in Fig. 8.

Interpretation of our findings is contingent on knowledge of the suite of measures that were implemented by the Egyptian authorities to control the epidemic. As soon as the first cholera cases were discovered in al-Qurayn, the village was cordoned off by police, and then guarded by the army. In echoes of the measures used since biblical times to control the spread of infectious diseases, various restrictive measures on the behaviour of the population were implemented across the country. ${ }^{38}$ These measures included: (i) the protection of water supplies, including the prohibition of washing and bathing in water courses and the 
chlorination of water supplies; (ii) the closure of public meeting places, including public swimming pools and public praying-places on the banks of the Nile; (iii) rubbish disposal and anti-fly measures, including the large-scale use of insecticides (DDT) to suppress the fly population; (iv) restrictions on public travel, including the control of river navigation and railway communications; and (v) vaccination, with the aim to inoculate the whole population as vaccine stocks became available from domestic and overseas sources. To these various measures can be added restrictions on the transport of fresh foodstuffs, restrictions on the movements of outbound pilgrims and the hospital isolation of cholera patients. ${ }^{39}$ Aspects of these control measures are illustrated in Figs. 9-12.

\section{FIGURE 9 NEAR HERE \\ FIGURE 10 NEAR HERE \\ FIGURE 11 NEAR HERE \\ FIGURE 12 NEAR HERE}

Particularly draconian restrictions were placed on public travel. These are described by Shousha:

Drastic measures were directed against the spread of infection from one zone to another. Thus, the isolation of infected provinces from the other provinces and governorates was carried out by the prohibition of any intercourse between their inhabitants. The latter were prohibited from proceeding from infected to uninfected localities unless they had been inoculated and six days had elapsed since the date of inoculation. Later it was found necessary to isolate Upper from Lower Egypt. ${ }^{40}$

In an effort to protect Cairo, military guards were placed at entry points into the city, and free passage was limited to those in possession of official vaccination certificates. Similar measures were implemented in other localities while, as noted above, river navigation and railway communications were strictly controlled. Such travel restrictions were utilised within the framework of a region-wide vaccination campaign: 
To separate completely Upper from Lower Egypt, trains were stopped for six days to allow for the vaccination of all inhabitants of Lower Egypt. After this interval, travelling was allowed, provided passengers were in possession of sixday-old inoculation certificates with photographs. These measures were maintained until free travelling was gradually authorized on the termination of inoculation of inhabitants. ${ }^{41}$

The impact of these efforts to isolate Lower and Upper Egypt can be seen in Fig. 7. In Fig. $7 \mathrm{~B}$, the infectives integral $I_{A}$ in Lower Egypt dropped in the third and fourth weeks of the epidemic as the initial control measures began to bite, before it increased again to a plateau in weeks six to nine; thereafter decline set in as the epidemic was brought under control. In Upper Egypt, the impact of the isolation policy, plus distance from Cairo, meant that cholera did not arrive until the fourth week. The integral then rose haltingly until the tenth week when decline set in. For the same reasons, in the first six weeks the shape of the curve for the spatial reproduction rate $R_{0 A}$ (Fig. 7C) echoes the infectives integral - falling at first in Lower Egypt before increasing; and, in Upper Egypt, showing a halting acceleration until week six.

Given the lengthy interval since the preceding epidemic of 1902, and that exposure to $V$. cholerae provides only short-term protection against reinfection, it is reasonable to assume that population susceptibility to cholera was more or less universal in Egypt in $1947 .{ }^{42}$ As such, and accepting that many mild and subclinical cases of disease are likely to have gone unrecorded, the 1947 epidemic had the potential to be of far greater proportions than the morbidity and mortality statistics would imply. The current outbreak of cholera in Haiti, associated with over 700,000 documented cases in a population of less than eleven million, provides some sense of the potential size of an epidemic when population susceptibility is universal and environmental conditions are favourable to $V$. cholerae transmission. ${ }^{43}$

In accounting for the differential rates of spatial propagation of the cholera wave in Lower and Upper Egypt ( $S_{A}, V_{L E}$ and $R_{0 A}$ in Figs. 7B and C), we note that our findings are consistent with the recognised tendency for the rate of transmission of an epidemic to 
decrease with distance in time and space from its origin. ${ }^{44}$ While this may reflect the gamut of control measures that were implemented to protect the population of Upper Egypt after the first recognition of the disease in the Nile Delta area, a range of factors associated with the social and physical environment may also have shaped the transmission of the disease in these areas of the country. ${ }^{45}$ To test one potential risk-modifier for exposure to V. cholerae, population density (persons per $\mathrm{km}^{2}$ ) in the provinces/governorates of Upper and Lower Egypt was entered as a predictor variable in a least squares multiple regression model in which the duration of the susceptibility period $S$ (Figure 6A) formed the response. Additional predictor variables included straight-line distance $(\mathrm{km})$ from the origin of the epidemic (alQurayn) and a dummy variable classification of provinces/governorates according to their location in Lower and Upper Egypt. Consistent with the swash-backwash advance and retreat of the epidemic in the Nile Valley, the regression model (overall fit: $R^{2}=0.51 ; F=5.24, P=$ $0.011)$ yielded a positive association for the distance variable $(t=2.03, P=0.061)$, but no association for the density variable $(t=0.38, P=0.708)$. Indeed, Shousha draws attention to the general tendency for epidemic activity to be focussed not in the densely populated towns and cities - where administrative and sanitary infrastructure was highest - but, rather, in villages and agricultural areas where such infrastructure was weakest. ${ }^{46}$ Under these circumstances, the role of rural areas as determinants of the disease patterns described in this paper would seem to merit additional analysis.

While various aspects of the official response to the cholera epidemic can be deciphered in Fig. 7, an additional facet of the swash-backwash analysis is evident: the rate of propagation of the infection wave was too rapid for complete spatial control to be effected by mass vaccination of the population in the fast-moving 'swash' stage of the epidemic. Prior to the onset of the epidemic, the regular daily output of cholera vaccine by the Vaccine and Serum Institute in Cairo was 40,000 $\mathrm{ml}$ (equivalent to 40,000 'usual' doses of vaccine) and this for a population of 19 million. ${ }^{47}$ Although the institute's output increased ten-fold during the first month of the epidemic, we noted in the introduction to this paper that international procurements and donations of vaccine were necessary to inoculate the whole population exposed to risk of infection. ${ }^{48}$ These supplies, however, arrived too late to permanently halt 
the spatial advance of cholera and, within five weeks of onset, most of the country had been entrained by the epidemic (Figs. 5 and 6). This is not to say that mass vaccination did not play an important role in the subsequent amelioration of the epidemic. ${ }^{49}$ The British Medical Journal, however, was suitably guarded in its judgement on the matter:

It is true that the over-all incidence [of cholera in Egypt] began to decline as soon as $80 \%$ of the population had been inoculated with anti-cholera vaccine, but whether this represents a causal relationship or merely the coincidence of inoculation and a spontaneous autumnal decline it is too early to say. ${ }^{50}$

One important limitation of the present analysis is the quality of the epidemiological data available for examination. While Shousha's report on the epidemic gives a total of $\sim 21,000$ cholera cases and $\sim 10,000$ cholera deaths, more recent estimates of cholera morbidity ( $\sim 35,000$ cases) and mortality ( 20,000 deaths) imply a 40-45 percent level of under-reporting in the official disease records. ${ }^{51}$ Recognising this concern, the swashbackwash model has provided us with a robust method for examining the 1947 cholera epidemic wave under conditions of uncertain data quality. The model is robust in the sense that it relies upon binary (start/end) data for an epidemic rather than complete counts of disease activity in the set of geographical units under examination. In judging the accuracy of the binary information, it is important to recognise that the majority of infections with $V$. cholerae tend to be subclinical or mild and, as such, the tails of an epidemic in a given geographical area are likely to go unrecorded. ${ }^{52}$ Under these circumstances, clinically recognised cases of cholera form a proxy for the epidemic start and end dates used in the present analysis.

\section{Conclusion}

This paper has shown how the linear constrictions imposed by the geography of the Nile Valley upon the distribution of settlements in Egypt served to shape the spatial diffusion of one of the twentieth century's great cholera outbreaks. Using a model designed to handle disease data of variable quality, the spatial diffusion of a cholera wave which arrived in the Nile Delta in September 1947 has been tracked as it progressed up the Nile Valley as far as 
Aswan, before retreating down the Nile like the backwash of a wave on a beach. In the event, a vigorous response by the Egyptian authorities succeeded in controlling the disease, leading Shousha to conclude that 'The final result of the 1947 epidemic was entirely contrary to what might have been expected in view of the easy and rapid means of communication now existing [in Egypt], ${ }^{53}$ But control was achieved only after virtually every province and governorate had been entrained by the epidemic and many thousands had died. Our results suggest that it was the rapid spatial velocity of the 'leading edge' of the infection wave, facilitated by the easy and rapid means of communication to which Shousha alludes, that permitted the epidemic to 'outrun' any early control efforts and to become seeded in the provinces and governorates of the country. 


\section{Acknowledgements}

We are grateful to the Editor and three anonymous referees for their helpful comments on an earlier version of this paper.

\section{Funding}

This work was supported by a grant from the Leverhulme Trust (RPG-2012-736). 
Table 1. Summary descriptors of the parameters of the swash-backwash model for cholera in Egypt, 1947

\section{Average velocity ratios}
$V_{L E}$
Dimensionless ratio with values in the range $[0,1]$. Relatively low / high values denote relatively slow / fast spreading infection waves.
$V_{F E}$ Dimensionless ratio with values in the range [0, 1]. Relatively low / high values denote relatively slow / fast retreating infection waves.

\section{Integrals}

$S_{A}$

Dimensionless number with values in the range [0, 1]. Relatively low / high values denote relatively fast / slow spreading infection waves.

Dimensionless number with values in the range [0, 1]. Relatively low / high

$I_{A} \quad$ values denote infection waves in which the rate of spatial retreat is relatively fast / slow compared with the rate of spatial advance.

$R_{A} \quad$ Dimensionless number with values in the range $[0,1]$. Relatively low / high values denote relatively slow / fast retreating infection waves.

\section{Spatial basic reproduction rate}
$R_{0 A} \quad$ Relatively low / high values denote relatively slow / fast spatial propagation of an infection wave.




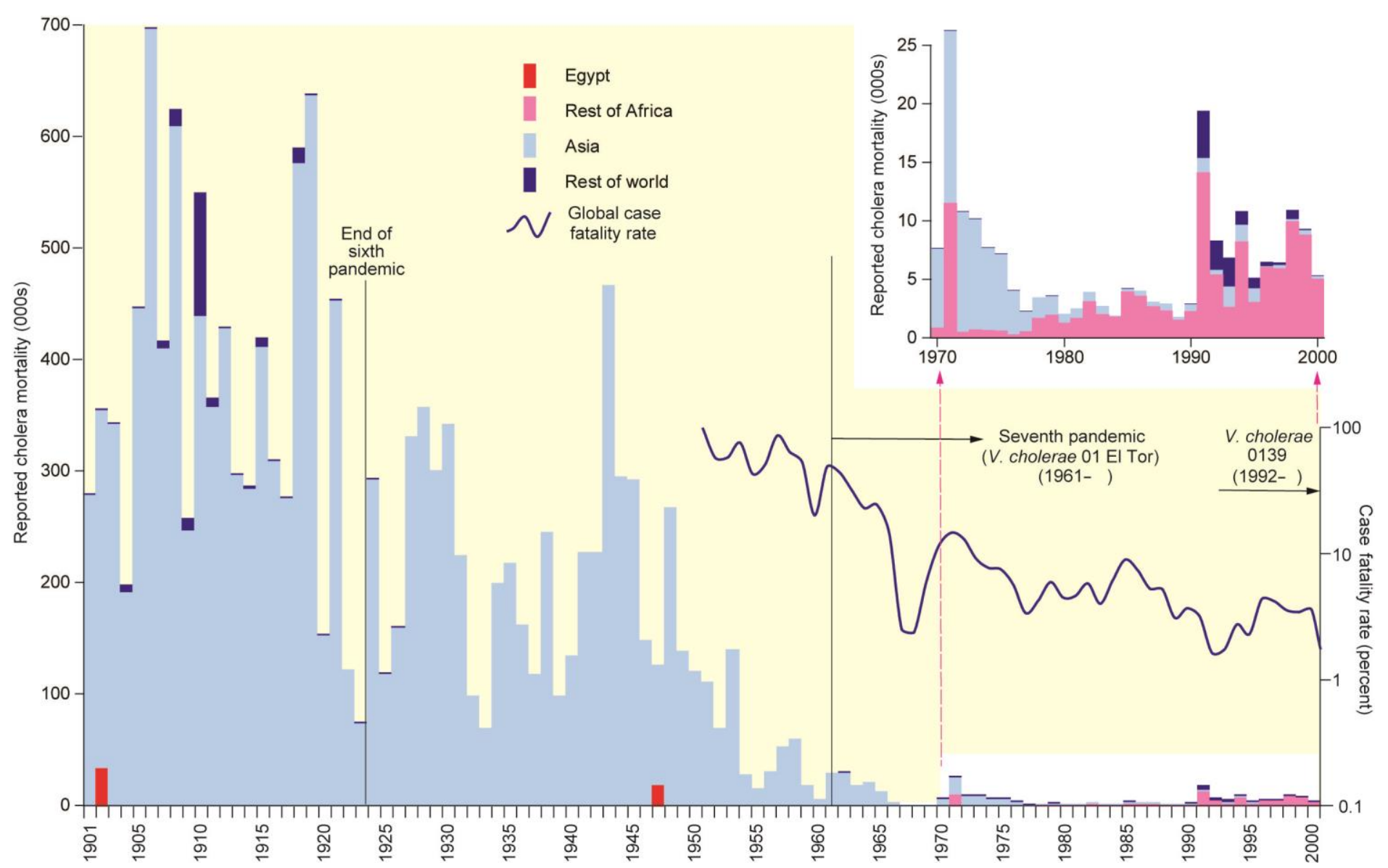

Fig. 1. Global cholera mortality trends in the twentieth century, 1901-2000. The cholera epidemics of 1902 and 1947 in Egypt are indicated. Sources: data from R. Pollitzer, W. Burrows and S. Swaroop, Cholera, Geneva, 1959, 58-59 (Table VI, 9); World Health Organization, World distribution of cholera endemicity, Epidemiological and Vital Statistics Report 5 (1952) 569-589 (Table 1, 570); and WHO Global Health Atlas, http://www.who.int/globalatlas/, last viewed 4 May 2016. 


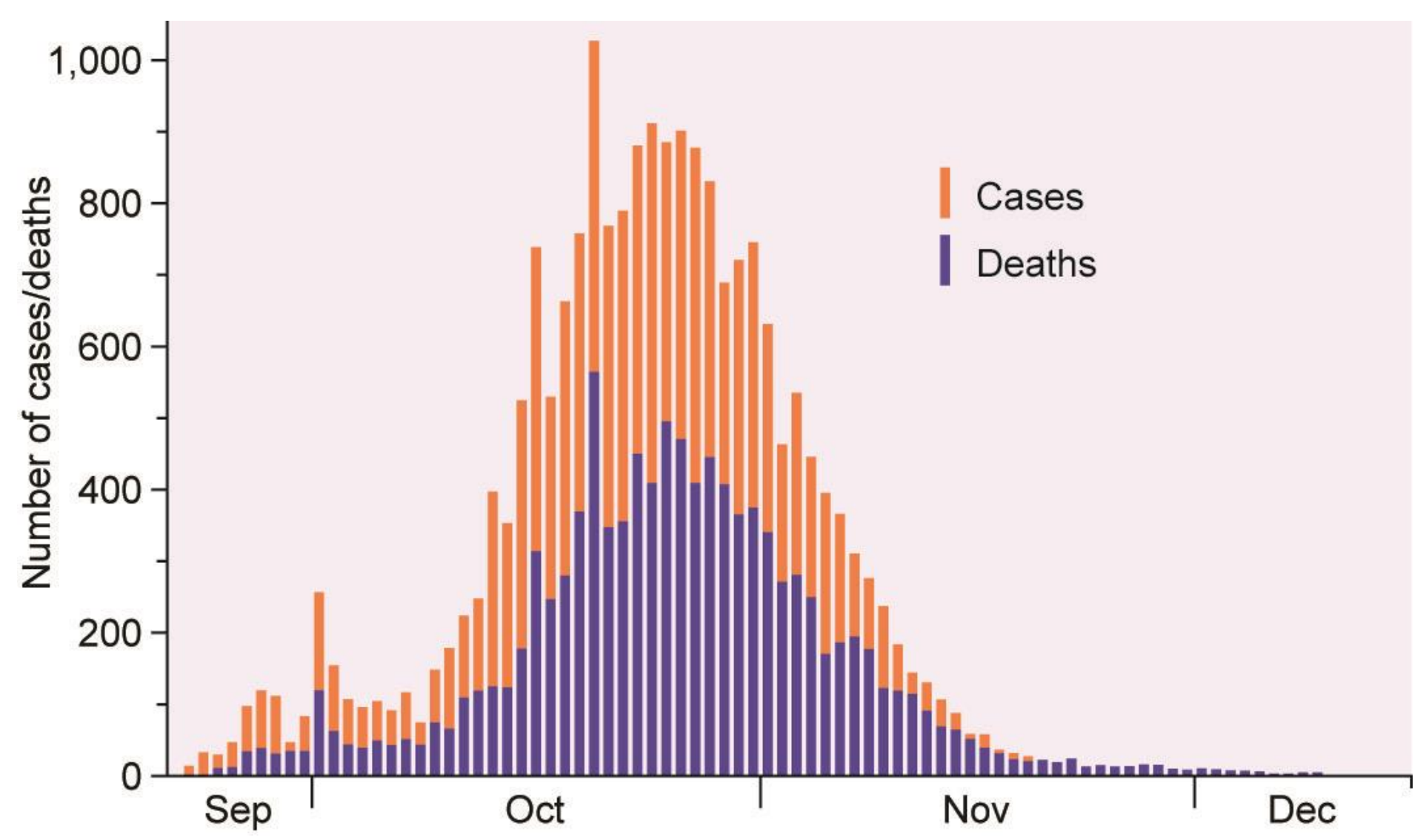

Fig. 2. Cholera epidemic in Egypt, 1947. Daily time series of reported cases and deaths, 21 September-15 December. Source: data from A.T. Shousha, Cholera epidemic in Egypt (1947): a preliminary report, Bulletin of the World Health Organization 1 (1948) 353-381 (Table 2, 372-373). 

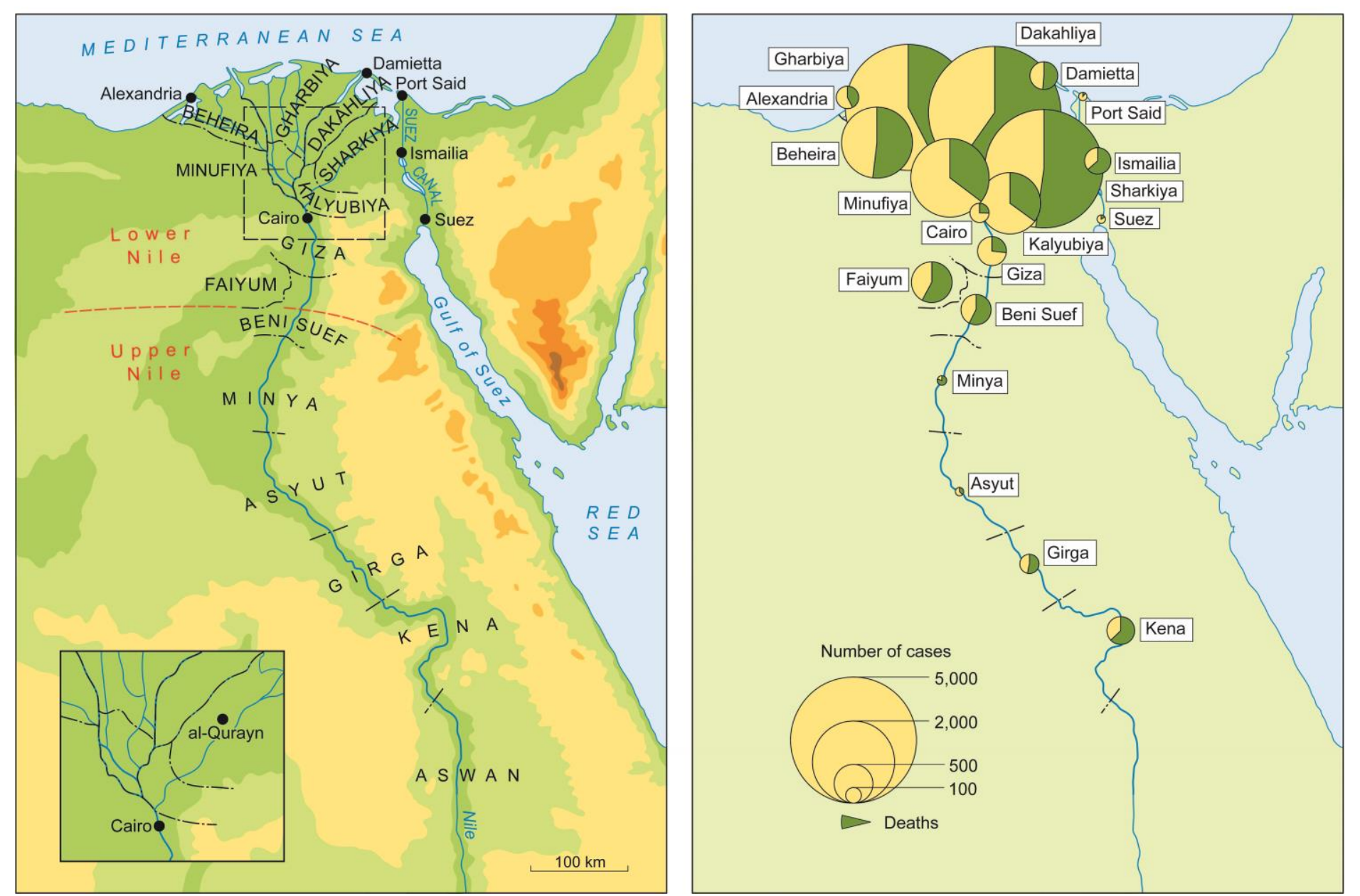

Fig. 3. Geographical distribution of cholera morbidity and mortality in Egypt, 1947. (Left) Location map of provinces and governorates with the dividing line between Upper and Lower Egypt marked. (Right) For each province/governorate, the proportional circles show the reported number of cholera cases and deaths. Source: data from A.T. Shousha, Cholera epidemic in Egypt (1947): a preliminary report, Bulletin of the World Health Organization 1 (1948) 353-381 (unnumbered figure, 357). 


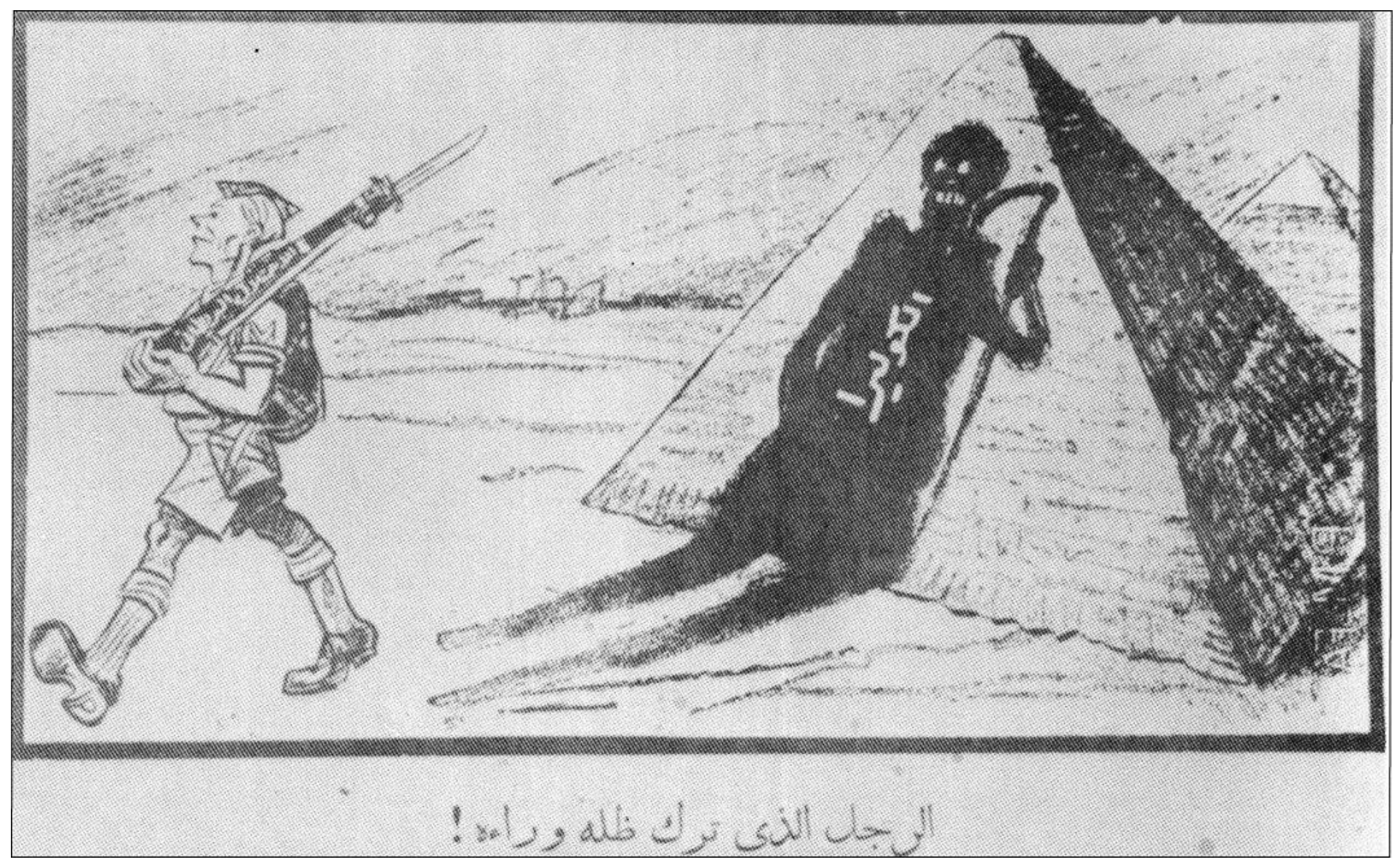

Fig. 4. Origin of the 1947 cholera outbreak in Egypt. The suspected local source of infection was the British air force base at Tal al-Kabir near al-Qurayn, and this was savagely depicted in the popular press. This newspaper cartoon is titled 'The man who left his shadow behind him'. The rifle reads 'British occupation' and the shadow is death named 'Cholera'. Source: Akhbar al-Yawm, 4 October 1947, reproduced with kind permission from N.E. Gallagher, Egypt's Other Wars: Epidemics and the Politics of Public Health, Syracuse, 1990, 140-159 (Figure 20, 143). 

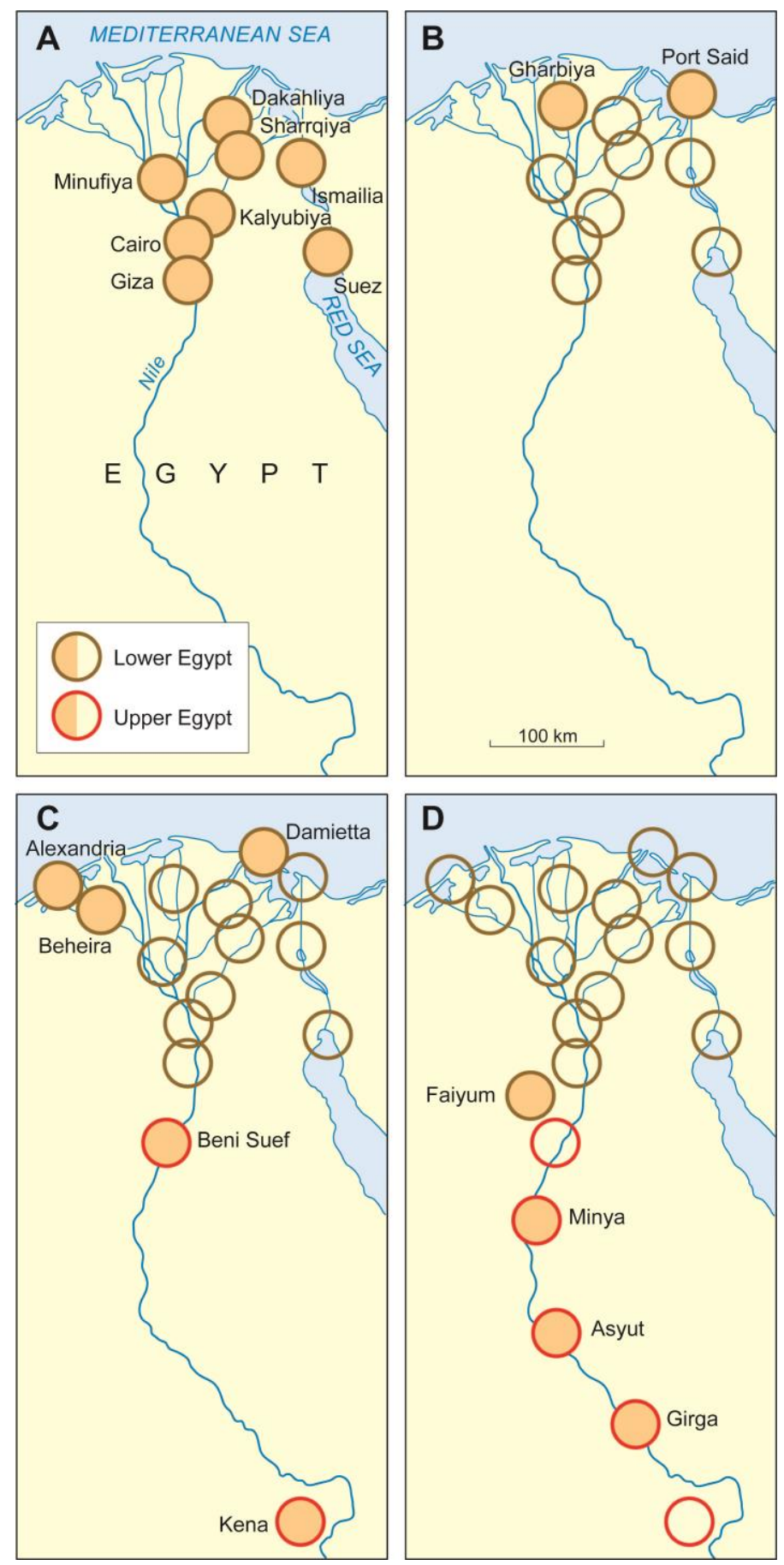

Fig. 5. Diffusion of cholera in the Nile Valley, September-October, 1947. The week of first report of cases by provinces and governorates is shown by coloured circles. The line colour of the circle perimeters indicates to which of the historical divisions of Egypt - Upper (red) or Lower (blue) - the province/governorate belongs. Provinces/governorates which appear on earlier maps in the sequence are plotted as open circles. (A) Week 39 (Sept). (B) Week 40 (Sept). (C) Weeks 41, 42 (Sept); Weeks 43-45 (Oct). 

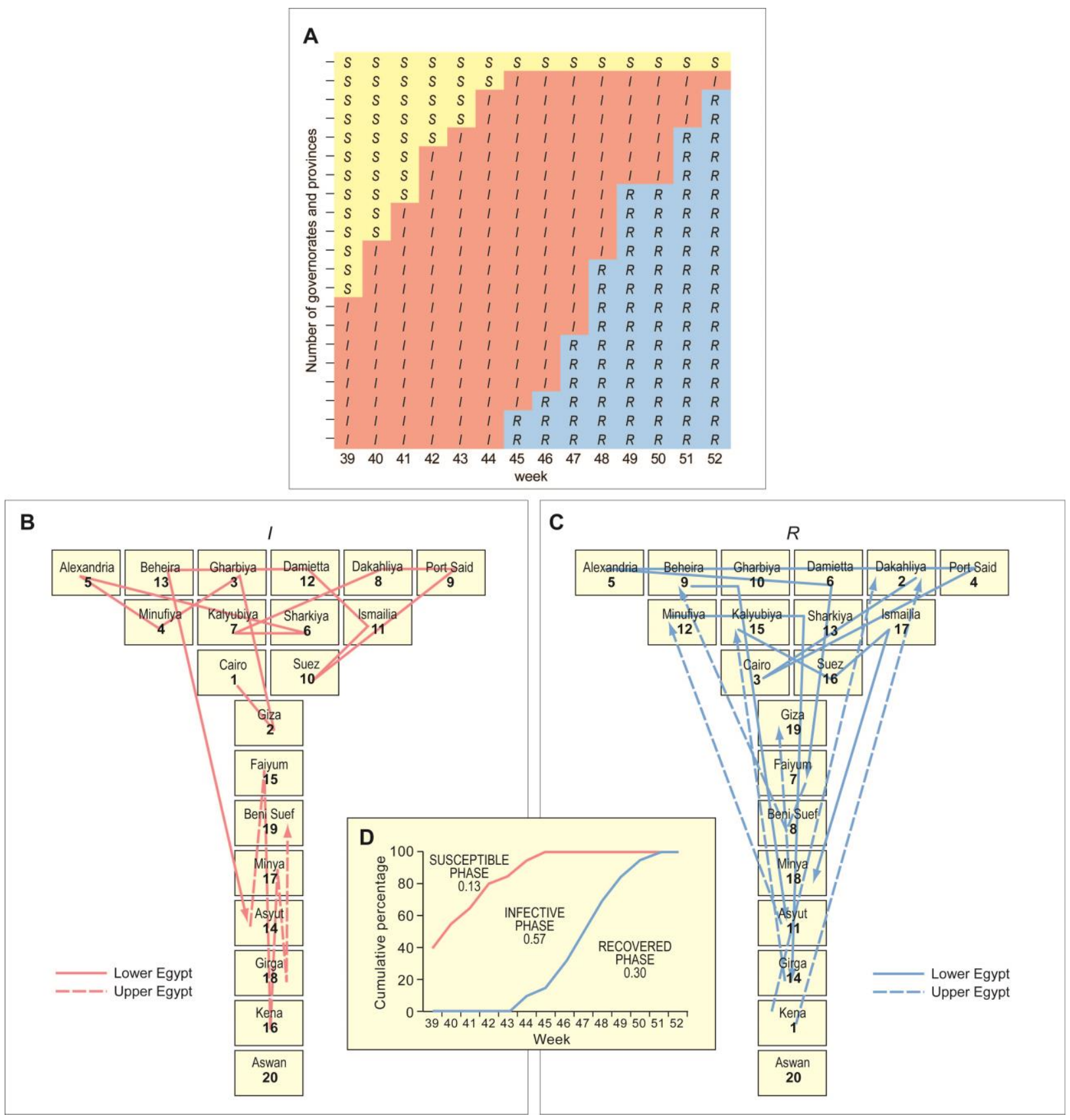

Fig. 6. Cholera in Egypt, 1947: application of the swash-backwash model to the epidemic. (A) Space-time map of the weeks of the epidemic for which each province/governorate had either not yet reported its first cholera case (susceptible, $S$ ), was reporting cases $(I)$, or had ceased reporting cases $(R)$. The number sequence and vectors on the accompanying flow maps plot the temporal sequence of province/governorate infection and recovery. (B) Infection. (C) Recovery. Arrow heads show the geographical trajectory of infection and recovery. (D) Epidemic integrals $S_{A}, I_{A}, R_{A}$ derived from (A) on the basis of the leading and following edge curves. Note that the collective area known as Frontiers has been excluded from maps $\mathrm{B}$ and $\mathrm{C}$. 

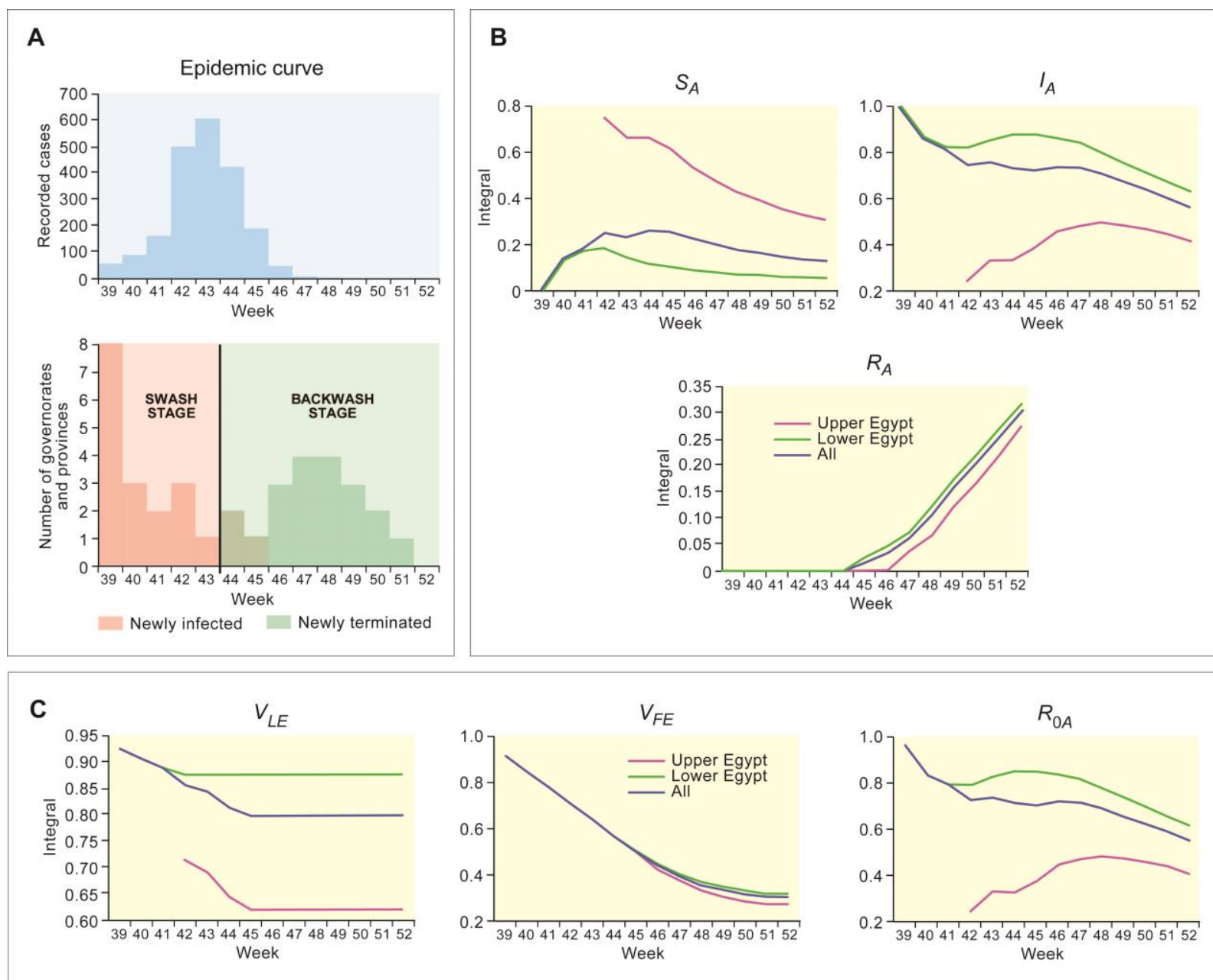

Fig. 7. Cholera in Egypt, 1947: epidemic stages and velocity. (A) Swash and backwash stages of the epidemic. The blue bar graph shows the epidemic curve as reported cases per week. In the associated combination chart, the bar charts plot, by epidemic week, the number of governorates that first notified ('newly infected') and last notified ('newly terminated') cases of cholera. The difference between the two time series defines the swash (pink bars; epidemic wave burgeoning) and backwash (green bars, epidemic wave retreating) stages of the epidemic. (B) Time trajectories of the $S, I$, and $R$ integrals over the duration of the epidemic are plotted for all provinces/governorates and for those comprising Lower and Upper Egypt. (C) Velocity of the leading $\left(V_{L E}\right)$ and following $\left(V_{F E}\right)$ edges in each week of the epidemic, and the spatial basic reproduction rate, $R_{0 A}$. 
A Case rate/100,000 population
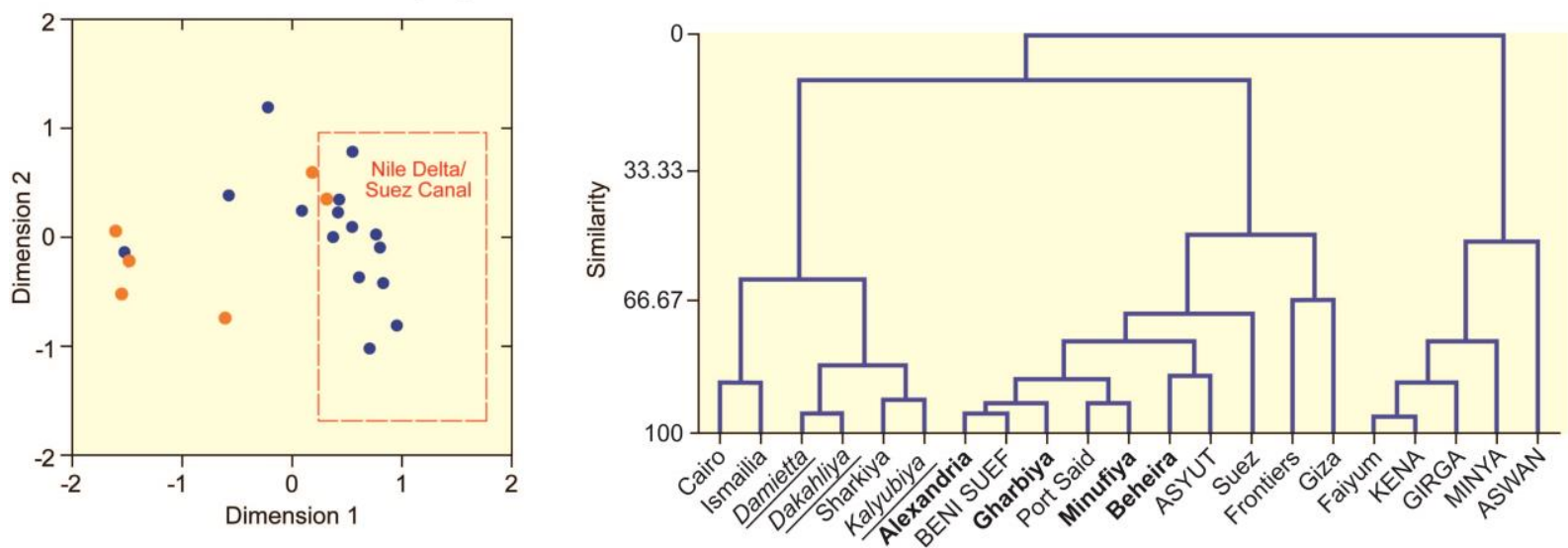

B Death rate/100,000 population

Governorates and provinces
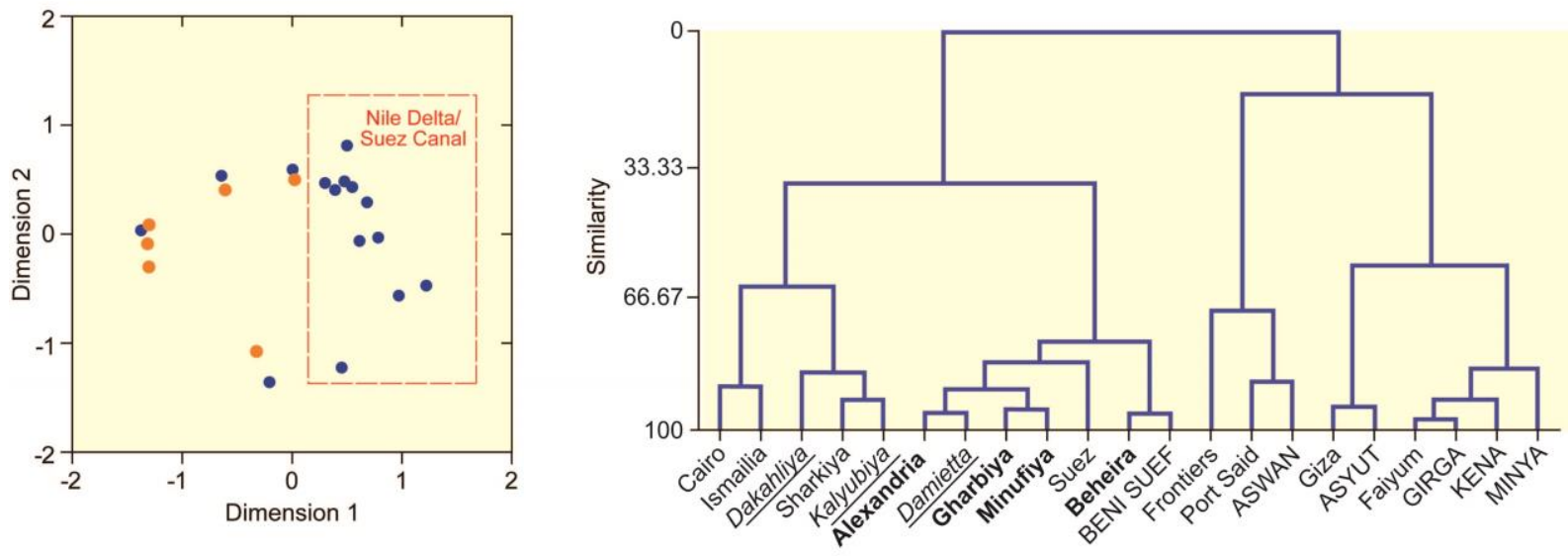

Governorates and provinces

Fig. 8. Egypt's MDS cholera spaces, 21 governorates and provinces. (A) Reported case rates per 100,000 population. (B) Reported death rates per 100,000 population. The left part of each diagram gives the MDS minimum stress configurations in two dimensions. Red circles denote Upper Nile provinces and governorates, blue circles those of the Lower Nile. Those in the Delta and Suez Canal areas are boxed. The right part of each diagram shows the clustering dendrogram where the naming of governorates and provinces is as follows: capitals - Upper Nile; underlined italic - East Nile Delta; bold - West Nile Delta. 

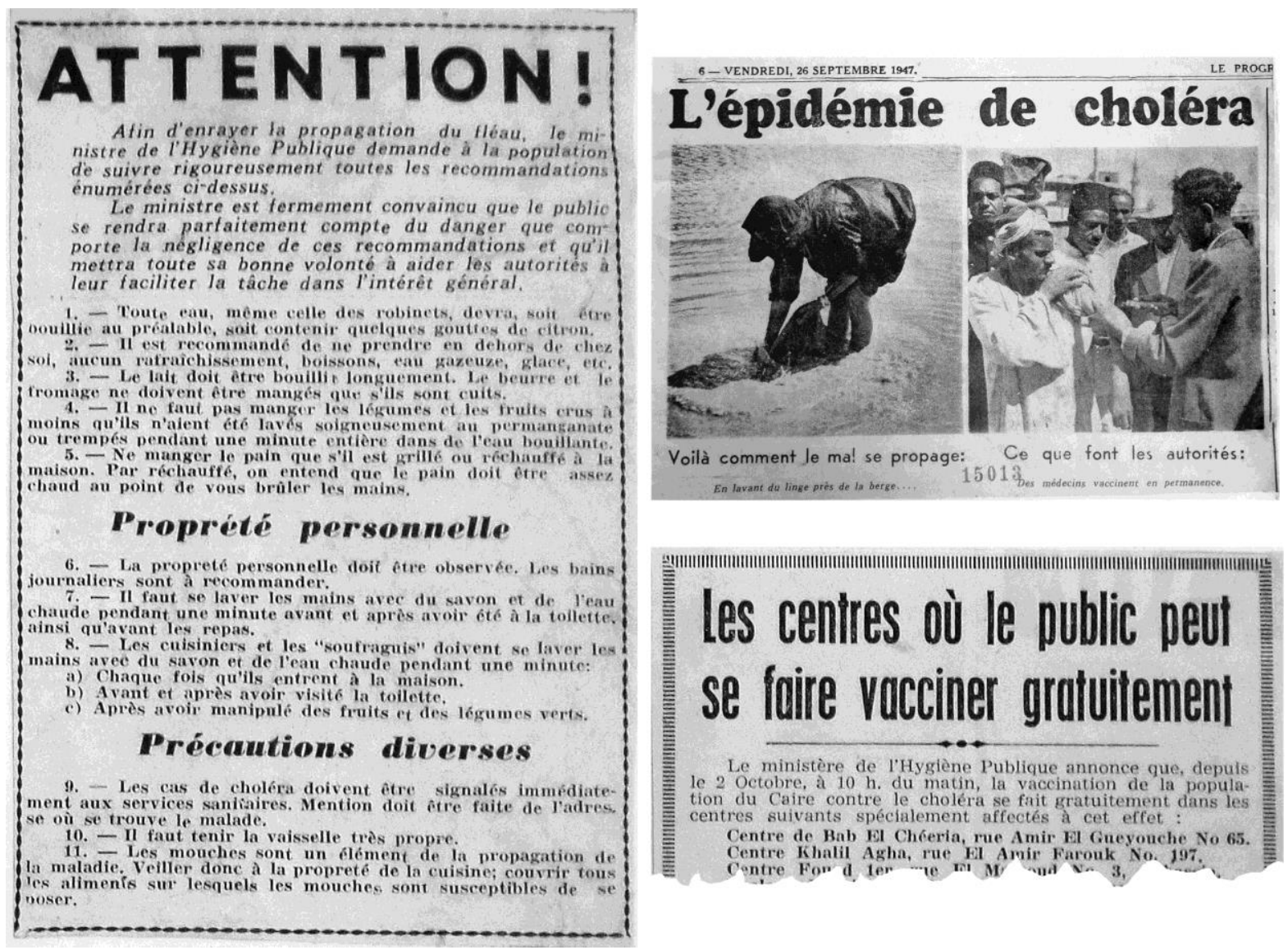

Fig. 9. Controlling the spread of cholera in Egypt during the 1947 outbreak. Newspaper cuttings describing the restrictions imposed by the government upon the day-to-day behaviour of the Egyptian people. Source: Archives of the International Committee of the Red Cross (ACICR), Geneva, Box CR233-22 (CR233/2, Commission médicale-maladies diverses/maladies infectieuses). 


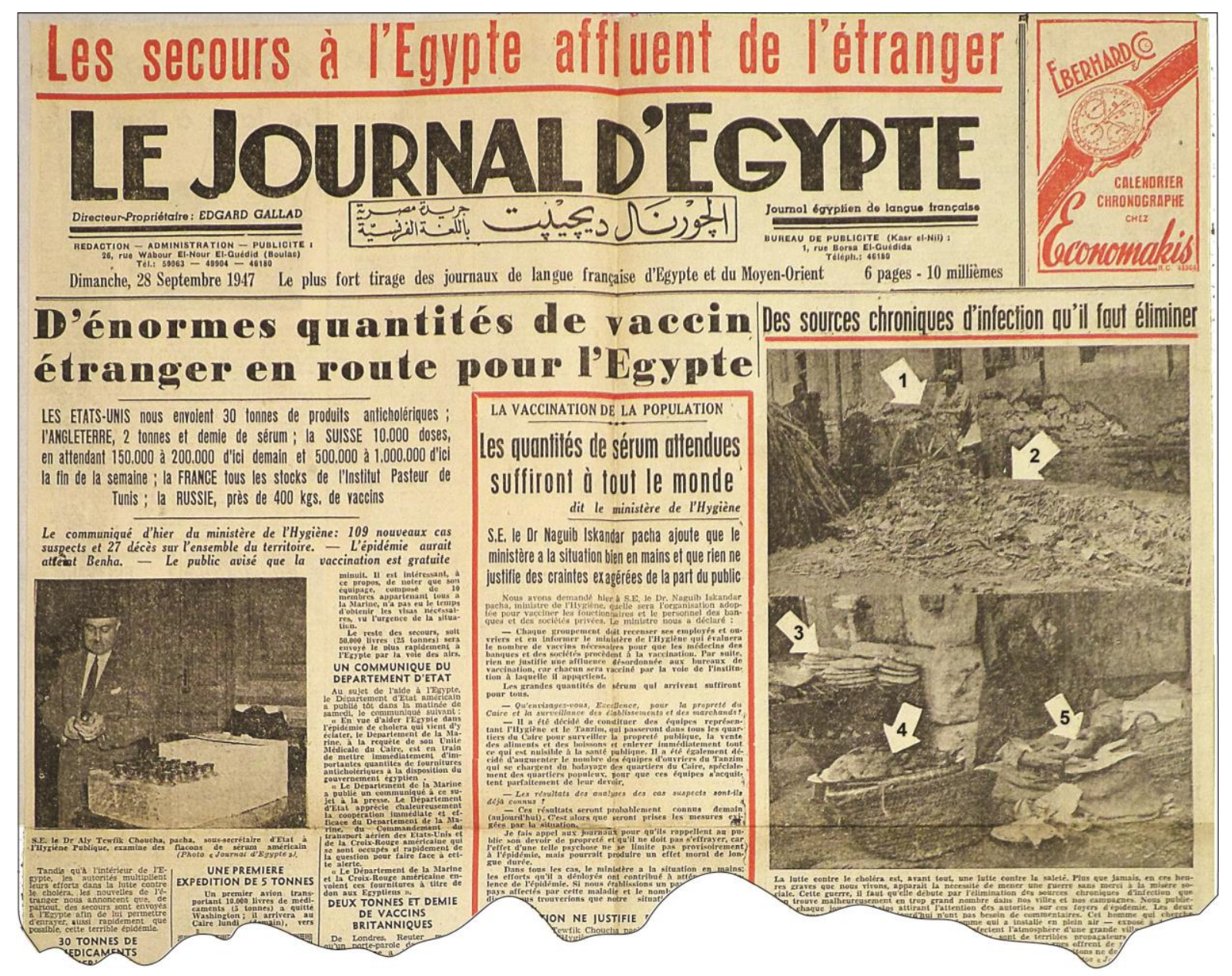

Fig. 10. Vaccinating the Egyptian population. Cutting from Le Journal d'Egypt 28

September giving (a) details of the deliveries of cholera vaccine from the United States, United Kingdom, Switzerland, France and Russia to permit mass vaccination of the Egyptian popualtion against cholera, and (b) possible sources of infection to be eradicated. Source: Archives of the International Committee of the Red Cross (ACICR), Geneva, Box CR233-22 (CR233/2, Commission médicale-maladies diverses/maladies infectieuses). 


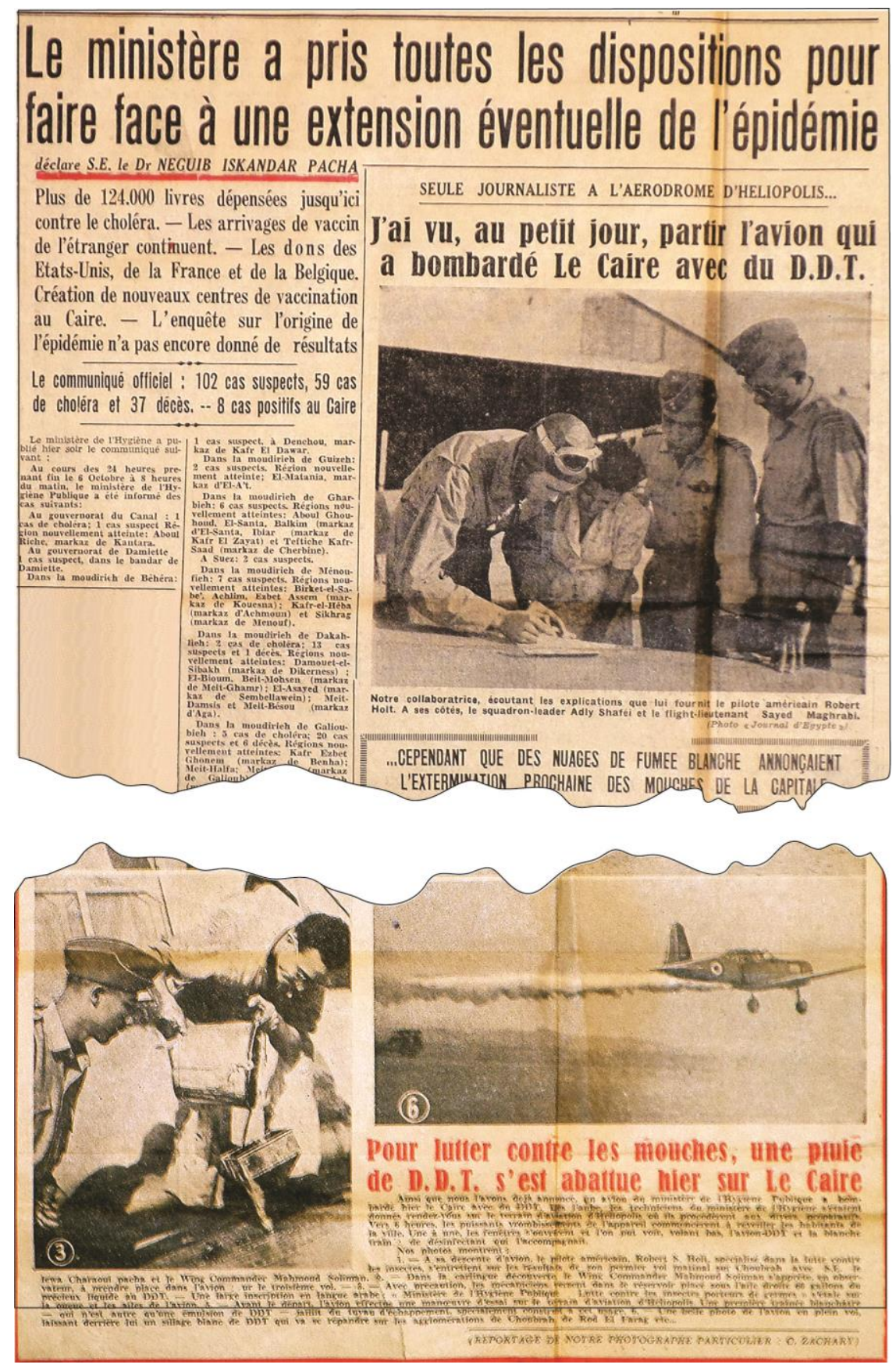

Fig. 11. DDT spraying of Cairo. Fear of vibrio transmission by flies led to an international effort to control the insects in many towns in the Nile Delta by DDT spraying of houses and mass aerial spraying with a 10 percent solution of DDT in kerosene liquid. This newspaper cutting from Le Journal d'Egypt informs its readers of the spraying programme. Source: Archives of the International Committee of the Red Cross (ACICR), Geneva, Box CR233-22 (CR233/2, Commission médicale-maladies diverses/maladies infectieuses). 


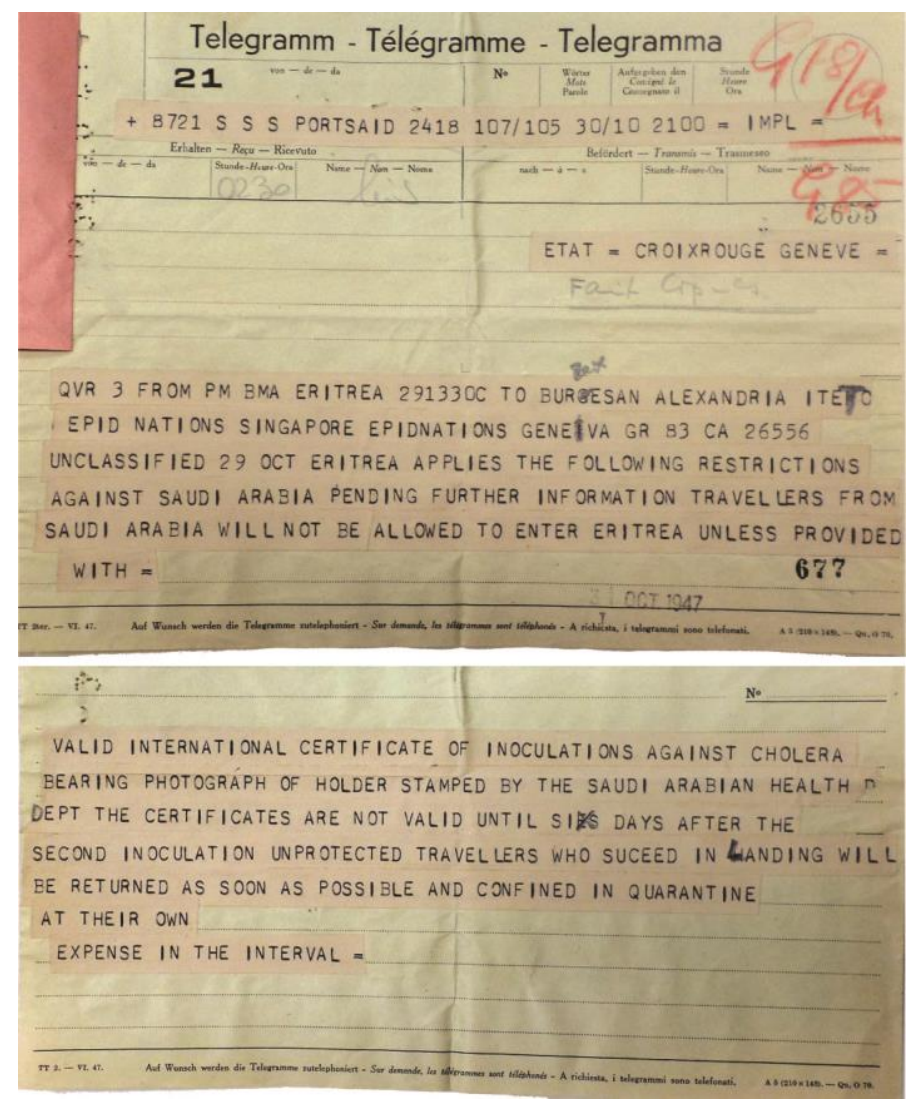

Fig. 12. Cholera and the Hajj. To mitigate the risk of entrainment of the cholera outbreak among pilgrims travelling to/from Mecca, international travel restrictions were imposed here between Saudi Arabia and Eritrea. As in Egypt, note the use of quarantine and a six-day window for vaccinations to take effect. Source: Archives of the International Committee of the Red Cross (ACICR), Geneva, Box CR233-22 (CR233/2, Commission médicale-maladies diverses/maladies infectieuses). 


\section{NOTES}

${ }^{1}$ World Health Organization, The First Ten Years of the World Health Organization, Geneva, $1958,60$.

2 Anonymous, Cholera in Egypt, British Medical Journal 1 (1948) 20.

${ }^{3}$ A.T. Shousha, Cholera epidemic in Egypt (1947): a preliminary report, Bulletin of the World Health Organization 1 (1948) 353-381.

${ }^{4}$ J. Farley, Brock Chisholm, the World Health Organization, and the Cold War, Vancouver, 2009, 51.

${ }^{5}$ Farley, Brock Chisholm, the World Health Organization, and the Cold War (note 4) 51-52.

${ }^{6}$ Shousha, Cholera epidemic in Egypt (1947): a preliminary report (note 3).

${ }^{7}$ Shousha, Cholera epidemic in Egypt (1947): a preliminary report (note 3) 372-373; M. Echenberg, Africa in the Time of Cholera: A History of Pandemics from 1815 to the Present, Cambridge, 2011, 80 .

${ }^{8}$ Shousha, Cholera epidemic in Egypt (1947): a preliminary report (note 3) 360.

${ }^{9}$ N.E. Gallagher, Egypt's Other Wars: Epidemics and the Politics of Public Health, Syracuse, 1990, 140-159; Echenberg, Africa in the Time of Cholera: A History of Pandemics from 1815 to the Present (note 7); R.F. Stock, Cholera in Africa: Diffusion of the Disease 1970-1975, with Particular Emphasis on West Africa. London, 1976, 19.

${ }^{10}$ Stock, Cholera in Africa: Diffusion of the Disease 1970-1975, with Particular Emphasis on West Africa (note 9).

${ }^{11}$ A.D. Cliff and P. Haggett, A swash-backwash model of the single epidemic wave, Journal of Geographical Systems 8 (2006) 227-252; M.R. Smallman-Raynor and A.D. Cliff, The geographical spread of the 1947 poliomyelitis epidemic in England and Wales: spatial wave propagation of an enigmatic epidemiological event, Journal of Historical Geography 40 (2013) 36-51.

12 D.L. Heymann (Ed.), Control of Communicable Diseases Manual, 20th Edition, Washington, D.C., 2015, 102-109.

${ }^{13}$ Heymann, Control of Communicable Diseases Manual (note 12) 104. 
${ }^{14}$ R. Pollitzer, W. Burrows and S. Swaroop, Cholera, Geneva, 1959, 17.

${ }^{15}$ E.H. Ackerknecht, History and Geography of the Most Important Diseases, Hafner, 1965, 23.

${ }^{16}$ Pollitzer, Burrows and Swaroop, Cholera (note 14).

17 Pollitzer, Burrows and Swaroop, Cholera (note 14); D. Barua and W.B. Greenough, Cholera, New York, 1992.

18 A. Cliff and M. Smallman-Raynor, Oxford Textbook of Infectious Disease Control. A Geographical Analysis from Medieval Quarantine to Global Eradication, Oxford, 2013, 41

${ }^{19}$ M. Smallman-Raynor, A.D. Cliff and A. Barford, Geographical perspectives on epidemic transmission of cholera in Haiti, October 2010-March 2013, Annals of the Association of American Geographers 105 (2015) 665-83.

${ }^{20}$ Shousha, Cholera epidemic in Egypt (1947): a preliminary report (note 3).

${ }^{21}$ Shousha, Cholera epidemic in Egypt (1947): a preliminary report (note 3) 354.

${ }^{22}$ Shousha, Cholera epidemic in Egypt (1947): a preliminary report (note 3) 372-373.

${ }^{23}$ Echenberg, Africa in the Time of Cholera: A History of Pandemics from 1815 to the Present (note 7) 80.

${ }^{24}$ Smallman-Raynor, Cliff and Barford, Geographical perspectives on epidemic transmission of cholera in Haiti, October 2010-March 2013 (note 19).

${ }^{25}$ Gallagher, Egypt's Other Wars: Epidemics and the Politics of Public Health (note 9).

${ }^{26}$ Gallagher, Egypt's Other Wars: Epidemics and the Politics of Public Health (note 9) 140159. The index case and the geographical origin will now probably never be known with certainty. Gallagher describes how the information disappeared into a morass of claim and counter claim between the British and the Egyptian governments, linked to a powerful political desire by the Egyptian people to be rid of the British occupation of their country. The mystery is deepened by missing papers in the UK National Archives relating to the origin of the epidemic. 
27 M.R. Smallman-Raynor, A.D. Cliff and H.J. Hooper, Atlas of Refugees, Displaced Populations and Communicable Disease. Decoding Global Geographical Patterns and Processes since 1901, Oxford (2016, in press).

${ }^{28}$ Shousha, Cholera epidemic in Egypt (1947): a preliminary report (note 3).

${ }^{29}$ Echenberg, Africa in the Time of Cholera: A History of Pandemics from 1815 to the Present (note 7) 79-80.

${ }^{30}$ Shousha, Cholera epidemic in Egypt (1947): a preliminary report (note 3) 372-373.

${ }^{31}$ A.D. Cliff and P. Haggett, A swash-backwash model of the single epidemic wave (note 11); M.R. Smallman-Raynor and A.D. Cliff, The geographical spread of the 1947 poliomyelitis epidemic in England and Wales: spatial wave propagation of an enigmatic epidemiological event (note 11); A.D. Cliff, P. Haggett and M.R. Smallman-Raynor, An exploratory method for estimating the changing speed of epidemic waves from historical data, International Journal of Epidemiology 37 (2008) 106-112.

${ }^{32}$ Cliff and Haggett, A swash-backwash model of the single epidemic wave (note 11).

${ }^{33}$ Smallman-Raynor and Cliff, The geographical spread of the 1947 poliomyelitis epidemic in England and Wales: spatial wave propagation of an enigmatic epidemiological event (note $11)$.

${ }^{34}$ A.D. Cliff, P. Haggett, M.R. Smallman-Raynor, D.F. Stroup and G.D. Williamson, The application of multidimensional scaling methods to epidemiological data, Statistical Methods in Medical Research 4 (1995) 102-23.

${ }^{35}$ For an overview of cluster analysis, see: B.S. Everitt, S. Landau, M. Leese and D. Stahl, Cluster Analysis, Chichester, 2011.

${ }^{36}$ Hierarchical clustering methods are described in Everitt, Landau, Leese and Stahl, Cluster Analysis (note 35) 71-110.

${ }^{37}$ Stock, Cholera in Africa: Diffusion of the Disease 197 -1975, with Particular Emphasis on West Africa (note 9). 
Cliff and Smallman-Raynor, Oxford Textbook of Infectious Disease Control. A Geographical Analysis from Medieval Quarantine to Global Eradication (note 18); Shousha, Cholera epidemic in Egypt (1947): a preliminary report (note 3).

${ }^{39}$ For an overview of the full range of control measures, see Shousha, Cholera epidemic in Egypt (1947): a preliminary report (note 3) 357-361.

${ }^{40}$ Shousha, Cholera epidemic in Egypt (1947): a preliminary report (note 3) 361.

${ }^{41}$ Shousha, Cholera epidemic in Egypt (1947): a preliminary report (note 3) 362.

42 Shousha, Cholera epidemic in Egypt (1947): a preliminary report (note 3); Heymann, Control of Communicable Diseases Manual (note 12).

${ }^{43}$ Smallman-Raynor, Cliff and Barford, Geographical perspectives on epidemic transmission of cholera in Haiti, October 2010-March 2013 (note 19).

${ }^{44}$ For a theoretical basis, see: D.G. Kendall, La propagation d'une épidémie au d'un bruit dans une population limitée, Publications de l'Institute de Statistique de l'Université de Paris 6 (1957) 307-311; A.D. Cliff, P. Haggett and M.R. Smallman-Raynor, Island Epidemics, Oxford, 2000, 120-123.

${ }^{45}$ Shousha, Cholera epidemic in Egypt (1947): a preliminary report (note 3).

${ }^{46}$ Shousha, Cholera epidemic in Egypt (1947): a preliminary report (note 3) 367-368.

${ }^{47}$ Shousha, Cholera epidemic in Egypt (1947): a preliminary report (note 3) 359-363.

${ }^{48}$ Shousha, Cholera epidemic in Egypt (1947): a preliminary report (note 3) 363.

${ }^{49}$ Shousha, Cholera epidemic in Egypt (1947): a preliminary report (note 3) 366.

${ }^{50}$ Anonymous, Cholera in Egypt (note 2).

51 Shousha, Cholera epidemic in Egypt (1947): a preliminary report (note 3) 372-373; Echenberg, Africa in the Time of Cholera: A History of Pandemics from 1815 to the Present (note 7) 80.

${ }^{52}$ Heymann, Control of Communicable Diseases Manual (note 12).

${ }^{53}$ Shousha, Cholera epidemic in Egypt (1947): a preliminary report (note 3) 366. 